\title{
Hepatitis E: An Underdiagnosed, Emerging Infection in Nonendemic Regions
}

\author{
Steven De Keukeleire and Marijke Reynders* \\ Department of Laboratory Medicine, AZ St-Jan Bruges, Bruges, Belgium
}

\begin{abstract}
Although hepatitis E virus (HEV) is the primary cause of enterically transmitted acute hepatitis and jaundice in developing countries, locally acquired HEV infections are increasing in nonendemic countries. As such, HEV is emerging as an underdiagnosed cause of infection. This report describes three clinically variable cases of HEV infection with unusual clinical presentations. These cases highlight the fact that HEV should be considered in the differential diagnosis of patients with unexplained hepatitis (acute or chronic) with or without extrahepatic manifestations. HEV should also be considered in patients with persistently elevated liver enzymes who have not travelled to known HEV-endemic regions. Lack of knowledge among physicians and an absence of standardized diagnostic tests may result in increased morbidity and mortality from HEV infection.

(c) 2015 The Second Affiliated Hospital of Chongqing Medical University. Published by XIA \& HE Publishing Ltd. All rights reserved.
\end{abstract}

\section{Introduction}

The hepatitis E virus (HEV) is a small, single-stranded, nonenveloped RNA virus, and is likely the most common cause of enterically transmitted acute hepatitis and jaundice worldwide. HEV has multiple nonhuman reservoirs, including pigs and rats. There are four known HEV genotypes: 1 (1a-1e) and $2(2 a-2 b)$ are human viruses, and $3(3 a-3 j)$ and $4(4 a-$ $4 \mathrm{~g})$ are zoonotic viruses. Four routes of HEV transmission have been described: fecal-oral and food-borne transmission, by transfusion of infected blood products or transplantation of infected tissues, and vertical (maternal-fetal) transmission.

Previous exposure to HEV can be determined by the presence of immunoglobulin (Ig) G anti-HEV antibodies. ${ }^{1-3}$ However, the incidence of HEV infection and associated sero-

Keywords: Autochthonous hepatitis E; Hepatitis E; HEV RNA; Polymerase chain reaction; Seroprevalence.

Abbreviations: ALT, alanine aminotransferase; CMV, cytomegalovirus; EBV, Epstein-Barr virus; GGT, gamma-glutamyltransferase; HAV, hepatitis A virus; HBV, hepatitis B virus; HCV, hepatitis C virus; HEV, Hepatitis E virus; HIV, human immunodeficiency virus; HSV, herpes simplex virus; Ig, immunoglobulin; VZV, varicella zoster virus.

Received: 30 October 2015; Revised: 24 November 2015; Accepted: 26 November 2015

DOI: $10.14218 /$ JCTH.2015.00039.

*Correspondence to: Marijke Reynders, Department of Laboratory Medicine, Clinical Microbiology, AZ Sint-Jan Bruges-Ostend, Ruddershove 10, Bruges 8000, Belgium. Tel: +32-5045-2603, Fax: +32-5045-2619, E-mail: marijke.reynders@azsintjan.be prevalence reflecting previous exposure to this Hepevirus are not known in Western countries. ${ }^{4-6} \mathrm{HEV}$ infection is known to cause self-limiting symptomatic or asymptomatic waterborne or enterically transmitted acute viral hepatitis. Nevertheless, $\mathrm{HEV}$ is clinically indistinguishable from acute hepatitis caused by other hepatotropic viruses. Moreover, only $\sim 35 \%$ of all HEV infections produce symptoms, and the percentage of asymptomatic infections is highest in children ( $<14$ years). ${ }^{7}$ Despite manifestation of acute infectious hepatitis (with jaundice, nausea, vomiting, pruritis, myalgia, abdominal pain, fever, gastroenteritis, or elevated transaminases), symptomatic infections may be misdiagnosed. Often, no etiology is found after ruling out alternative causal agents, such as hepatitis $A, B$, and $C$ viruses (HAV, HBV, and HCV, respectively), human immunodeficiency virus (HIV), cytomegalovirus (CMV), Epstein-Barr virus (EBV), herpes simplex virus (HSV), parvovirus B19, Toxoplasma gondii, alcohol, and toxic- and drug-related causes. This report describes three cases of HEV infection with differing clinical symptoms, highlighting the fact that HEV likely represents an underdiagnosed and emerging cause of hepatitis.

\section{Case reports}

\section{Patient 1}

In December 2009, a 32-year-old Caucasian woman was diagnosed with acute myeloid leukemia $[\mathrm{t}(11 ; 19)]$. She had no history of recent travel, contact with animals, or consumption of venison, liver sausage, or offal. She received a blood transfusion prior to undergoing consecutive cycles of chemotherapy. In February 2010, she underwent hematopoietic stem cell transplantation with cells obtained from her brother. A standard screening protocol performed prior to the transplantation showed she was seropositive for HSV, CMV, varicella zoster virus (VZV), EBV, and $T$. gondii. Further virologic investigation revealed vaccine-derived immunity to HBV, but no evidence of HAV, HCV, or HIV. One month posttransplantation, in March 2010, CMV reactivated and was treated with consecutive antiviral agents (valacyclovir, foscavir, ganciclovir, and cidofovir). In May 2010, pathologic laboratory values were obtained for alanine aminotransferase (ALT; $544 \mathrm{IU} / \mathrm{L})$, aspartate aminotransferase (259 IU/L), and gamma-glutamyltransferase (GGT; $92 \mathrm{IU} / \mathrm{L}$ ). Findings from an abdominal ultrasound were negative. She underwent a liver biopsy, with histologic findings compatible with acute viral hepatitis. In September 2010, she developed CMV-ventriculitis, which was confirmed by magnetic resonance imaging and presence of viral DNA in cerebrospinal fluid, and was successfully treated with ganciclovir plus cidofovir. 
De Keukeleire S. et al: Hepatitis E: An underdiagnosed, emerging infection

Liver function tests remained abnormal, and in November 2010, a serum test for HEV RNA was conducted, revealing a high viral load $\left(>5 \times 10^{6}\right.$ copies $/ \mathrm{mL}$ ). Thus, a diagnosis of HEV infection (genotype $3 f$ ) was made, and the patient was treated with ribavirin monotherapy. Serologic HEV testing (RecomWell Hepatitis IgG/IgM; Mikrogen $\mathrm{GmbH}$, Neuried, Germany) remained negative. Although no serum samples were available to evaluate pretransplant serostatus, the hematopoietic stem cell donor was HEV-seronegative. The HEV viraemia remained stable $\left(5 \times 10^{6}\right.$ copies $\left./ \mathrm{mL}\right)$ for several months. In February 2011, Aspergillus pneumonia with pleural effusion appeared, without hepatic involvement, which was treated with caspofungin plus amphotericin B. One week later, the patient became extremely confused and suffered from dizziness and reduced consciousness. Examination of a plasma sample showed 2,565 copies/mL of adenovirus in addition to the persistent, high HEV viral load $\left(5 \times 10^{6}\right.$ copies $/ \mathrm{mL}$ ), and a sample of cerebral spinal fluid was positive for HEV RNA (3,700 copies/mL); CMV, adenovirus, EBV, and Toxoplasma were excluded.

The patient died in March 2011 due to multiple organ failure. Based on the chronic (>3 months), high-level detection of HEV RNA and no reported recent travel to endemic areas, an autochthonous, chronic HEV infection was most likely. An alternative origin of infection, such as the ingestion of contaminated food during the post-transplant period or transplantation of contaminated cells or blood products could not fully be excluded. Furthermore, no remaining stem cell preparation (prepared in an external laboratory) could be tested for the presence of HEV RNA.

\section{Patient 2}

In March 2013, a 62-year-old Bulgarian man was admitted with a provisional diagnosis of acute hepatitis based on a preexisting chronic HBV infection (inactive HBV carrier state: positive for $\mathrm{HB}$ surface antigen, core antibody, and envelope antibody; negative for $\mathrm{HB}$ envelope antigen and surface antibody with existing cirrhosis and ascites). One day prior to admission, the patient suffered from an acute onset of progressive, worsening upper abdominal discomfort, frequent episodes of diarrhea (2-3 times/day), loss of appetite, and fulminant jaundice without systemic symptoms. Laboratory results revealed elevated levels of aspartate aminotransferase (2,452 IU/L), ALT (1,161 IU/L), and GGT (301 $\mathrm{IU} / \mathrm{L})$, with high total bilirubin $(392 \mu \mathrm{mol} / \mathrm{L}$; direct bilirubin level: $345 \mu \mathrm{mol} / \mathrm{L})$, and thrombocytopenia $\left(119 \times 10^{6} / \mathrm{L}\right)$; Creactive protein was markedly elevated $(41.6 \mathrm{mg} / \mathrm{L})$. HAV, $\mathrm{HCV}$, and HIV were excluded. Serologic markers of previous CMV, EBV, and T. gondii infections were positive. Abdominal ultrasound showed known macronodular liver cirrhosis with manifest splenorenal shunting and splenomegaly. Anti-HEV IgM $(165.9 \mathrm{IU} / \mathrm{mL})$ and IgG $(212.6 \mathrm{IU} / \mathrm{mL})$ antibodies were positive. To exclude acute HBV-associated liver failure, polymerase chain reaction testing was performed using a commercially available kit (Abbott Laboratories, USA) to detect HBVDNA $(<12.5 \mathrm{IU} / \mathrm{mL})$. HEV RNA was detected $\left(9.76 \times 10^{5}\right.$ copies $/ \mathrm{mL}$ ), and sequencing revealed HEV genotype 3f. Due to the severity of his condition, the patient was immediately transferred and underwent a high-urgency liver transplantation (CMV-positive transplant). The patient experienced the following complications: an ischemic cerebrovascular accident, acute renal failure, corticoid-induced diabetes mellitus, and
CMV reactivation one month post-transplant (plasma viral load: 1,493 IU/L).

In September 2013, the patient died as a result of cardiac deterioration. A possible route of transmission could not be proven. However, there was a history of recent consumption of insufficiently heated pork sausage. Furthermore, the patient was at increased risk of developing severe complications due to the preexisting chronic liver disease.

\section{Patient 3}

In June 2014, a 66-year-old Caucasian man was admitted for investigation of bilateral shoulder pain associated with remarkably dark urine. He revealed a ten-day history of general malaise, loss of appetite, anorexia, and an influenza-like syndrome with diffuse myalgia. The shoulder pain was evolutive, followed by progressive neurologic complaints, including muscular weakness and loss of force in the left hand accompanied by paresthesia of the fingers and dysesthesia of the palm of the hand. The patient reported a recent trip to Southeast Asia. Magnetic resonance imaging of the head and cervical spine showed no abnormalities, as did computed tomography of the thorax and abdomen. Liver function tests showed elevated ALT (188 IU/L), alkaline phosphatase (159 $\mathrm{IU} / \mathrm{L})$, total bilirubin $(29.1 \mu \mathrm{mol} / \mathrm{L})$, and GGT (814 IU/L). Serology was negative for CMV, EBV, HIV, HSV, VZV, T. gondii, parvovirus B19, and Coxiella burnetii. Serology for HBV was weakly positive for the core antibody (signal-to-cutoff ratio: 9.19), and negative for surface and envelope antigens and antibodies. Polymerase chain reaction for HBV was negative. Anti-HEV IgG (174 IU/mL) and IgM (137 IU/mL) were positive, although no serum HEV RNA could be detected at the time of diagnosis. Immunoblot analysis (RecomLine Hepatitis E IgG/IgM; Mikrogen $\mathrm{GmbH}$ ) confirmed the presence of the antibody with high suspicion of a genotype 3 infection (no bands for genotype 1 visible). It is likely that crossreactivity with acute $\mathrm{HEV}$ infection lead to a false-positive HB core antibody reactivity, which became undetectable during the convalescent phase of HEV infection. Electromyography confirmed bilateral (idiopathic) brachial plexopathy suggestive of Parsonage-Turner syndrome. Therefore, the patient was diagnosed with an acute HEV infection triggering a bilateral Parsonage-Turner syndrome.

The patient was managed conservatively with physical therapy and nonsteroidal anti-inflammatory drugs and opiates for pain relief, without any antiviral therapy. Neurologic signs and symptoms improved within two months, and laboratory parameters returned to values within the physiologic reference range within one month. The patient recovered completely after eight months of intensive revalidation exercise.

\section{Discussion}

HEV has typically been considered as an imported, travelassociated disease. However, sufficient evidence has been gathered indicating that it is neither rare nor limited to developing countries. The diagnosis of HEV infection in immunocompetent individuals is based upon the detection of specific anti-HEV IgG and/or IgM antibodies in serum. AntiHEV IgM is detectable from the fourth day after disease onset and persists for up to 3-5 months, whereas anti-HEV IgG appears shortly after IgM with a peak concentration at approximately four weeks after onset of symptoms and 
persisting for 1-14 years. ${ }^{8}$ Although diagnostic tests have improved in recent years, the analytical performance of anti-HEV IgG assays remains controversial. ${ }^{9}$ International standardization should be encouraged. Although the detection of HEV RNA in serum or stool is the "gold standard" for confirmation of an acute or chronic active infection, negative HEV serology does not rule out the diagnosis.

The actual HEV seroprevalence in our region, West-Flanders, Belgium, was determined in 2012 by examining sera from 200 healthy blood donors, with detectable IgM and IgG antibodies in $4 \%$ and $12 \%$, respectively. These findings are comparable with the data obtained by Van Hoecke et $a{ }^{4}$ and Echevarria. ${ }^{5}$ Attempts to ascertain the HEV prevalence are hampered by unresolved technical issues (e.g., collaborating laboratories and lack of international standards in serology). ${ }^{5}$

As observed in the first patient, most solid organ transplant and hematopoietic stem cell transplant patients have no clear symptoms when infected with $\mathrm{HEV}$, and very few present with jaundice. Liver abnormalities detected by blood tests are usually very modest (typically ALT is $\sim 300 \mathrm{IU} / \mathrm{L}$ ) with no detectable anti-HEV IgG or IgM antibodies, and seroconversion stay absent after infection. ${ }^{10}$ Immunocompromised patients should be screened for HEV antibodies and RNA not only prior to transplantation, but also post-transplantation and during episodes of liver enzyme abnormalities. ${ }^{11}$ As observed in the third patient, neurologic signs and symptoms are emerging as potential extrahepatic manifestation of HEV genotype 3 infection. Associations between acute HEV infection and neurologic manifestations (e.g., Guillain-Barré syndrome, neuralgic amyotrophy, acute transverse myelitis, and encephalopathy) have been described. ${ }^{12-14}$ In patients with neurologic disorders, especially those with peripheral (brachial) nerve involvement and liver abnormalities, the possibility of HEV infection should be excluded along with several other microorganisms linked to Parsonage-Turner syndrome (including EBV, HSV, CMV, VZV, parvovirus B19, HIV, HBV, coxsackie $B$ virus, West Nile virus, and dengue).

Most cases of acute HEV infections are self-limiting and require no treatment, though clinical outcomes of HEV infection are quite diverse. Evolution of acute HEV infection to chronicity may occur, indicated by persistently elevated liver enzymes and detectable serum HEV RNA for at least three months after the acute episode, along with histologic findings of chronic hepatitis. ${ }^{12}$ In relation to the second patient described in this report, the mortality of an acute HEV superinfection with underlying chronic liver disease is approximately $70 \% .{ }^{1}$ In patients with a preexisting chronic liver disease, most deaths from HEV genotype 3 infections are caused by acute or subacute fulminant liver failure. ${ }^{1}$ Furthermore, alcohol consumption, HBV positivity, professional exposure to animals, older age, hemodialysis, immunodepression (solid organ transplantation, HIV, hematologic malignancies), and iv-drug use have been reported as risk factors. ${ }^{15-17}$

The high rate of asymptomatic HEV infections has raised concerns about infection by blood donation. ${ }^{18-20}$ Donated blood is currently not routinely tested for the presence of HEV RNA in Belgium. Transmission of HEV infection associated with transfusion might, therefore, be underdiagnosed. This may be of minor concern to immunocompetent patients, however, it is a clinically significant risk in immunocompromised patients. As fecal oral transmission is the predominant mode of HEV transmission in endemic regions, reducing $\mathrm{HEV}$ exposure forms the keystone in infection prevention.
In nonendemic countries, prevention is more complex, because several possible infection routes exist. Hand hygiene is probably the best preventive action. As occasional cases of HEV infection appear to be acquired by a zoonotic route, other preventive measures (e.g., adequate heating procedures for porcine and boar/deer meat) are also useful. Screening of donated blood is one way to prevent transmission through blood products, however its cost-effectiveness has not yet been determined. ${ }^{15}$

\section{Conclusions}

These distinct clinical cases highlight the fact that HEV should be considered in the differential diagnosis of patients with unexplained hepatic disturbances (acute or chronic) with or without extrahepatic manifestations. Although first truly identified in 1983, our knowledge about HEV, the prevalence, mode of infection, and potential treatment possibilities has only evolved significantly over the last few years. The lack of well-established diagnostic criteria and the absence of specific antiviral drugs are two points to consider in the work-up of patients with disturbed liver enzymes. In actuality, a considerable number of autochthonous infections likely remains undiagnosed for all the reasons discussed above.

\section{Acknowledgments}

We would like to thank all technicians of the laboratory medicine (infectious serology and molecular biology) of AZ St-Jan Bruges for their work and dedication.

\section{Conflict of interest}

None.

\section{Author contributions}

Data analysis, research of the background literature on the cases, writing the first draft (SDK); discussions and analysis of the cases, writing the manuscript (MR).

\section{References}

[1] Kamar N, Bendall R, Legrand-Abravanel F, Xia NS, Ijaz S, Izopet J, et al. Hepatitis E. Lancet 2012;379:2477-2488. doi: 10.1016/S0140-6736(11) 61849-7.

[2] Hoofnagle JH, Nelson KE, Purcell RH. Hepatitis E. N Engl J Med 2012;367: 1237-1244. doi: 10.1056/NEJMra1204512.

[3] Aggarwal R, Naik S. Epidemiology of hepatitis E: Current status. J Gastroenterol Hepatol 2009;24:1484-1493. doi: 10.1111/j.1440-1746.2009. 05933.x.

[4] Van Hoecke F, Van Maerken T, De Boulle M, Geerts A, Vlieberghe V, Colle I, et al. Hepatitis E seroprevalence in East and West Flanders, Belgium. Acta Gastroenterol Belg 2012;75:322-324.

[5] Echevarria JM. Autochtonous Hepatitis E Virus Infection in Europe: A Matter of Concern for Public Health? J Clin Transl Hepatol 2014;2:7-14. doi: 10.14218/ JCTH.2013.00027.

[6] Echevarria JM. Light and Darkness: Prevalence of Hepatitis E Virus Infection among the General Population. Scientifica 2014;2014:481016. doi: 10.1155/ 2014/481016.

[7] Dalton HR, Hazeldine S, Banks M, Ijaz S, Bendall R. Locally acquired hepatitis $E$ in chronic liver disease. Lancet 2007;369:1260. doi: 10.1016/S0140-6736 (07)60595-9.

[8] Dawson G], Mushahwar IK, Chau KH, Gitnick GL. Detection of long-lasting antibody to hepatitis E virus in a US traveller to Pakistan. Lancet 1992;340: 426-427. 
De Keukeleire S. et al: Hepatitis E: An underdiagnosed, emerging infection

[9] Avellon A, Morago L, Garcia-Galera M, Munoz M, Echevarria JM. Comparative Sensitivity of Commercial Tests for Hepatitis E Genotype 3 Virus Antibody Detection. J Med Virol 2015;87:1934-1939. doi: 10.1002/jmv.24251.

[10] Legrand-Abravanel F, Kamar N, Sandres-Saune K, Lhomme S, Mansuy JM, Muscari $F$, et al. Hepatitis $E$ virus infection without reactivation in solid-organ transplant recipients, France. Emerg Infect Dis 2011;17:30-37. doi: 10.3201/eid1701.100527.

[11] Gauss A, Wenzel J], Flechtenmacher C, Navid MH, Eisenbach C, Jilg W, et al. Chronic hepatitis $\mathrm{E}$ virus infection in a patient with leukemia and elevated transaminases: a case report. J Med Case Rep 2012;6:6334. doi: 10.1186/ 1752-1947-6-334.

[12] Ahmed A, Ali IA, Ghazal H, Fazili J, Nusrat S. Mystery of hepatitis E virus: recent advances in its diagnosis and management. Int J Hepatol 2015;2015: 872431. doi: 10.1155/2015/872431.

[13] Wu X, Liu K, Zhang HL. Guillain-Barré syndrome and encephalitis/encephalopathy associated with acute severe hepatitis E infection. Neurol Sci 2014; 36:165-166. doi: 10.1007/s10072-014-1854-x.

[14] Deroux A, Brion JP, Hyerle L, Belbezier A, Vaillant M, Mosnier E, et al. Association between hepatitis $E$ and neurological disorders: two case studies and literature review. J Clin Virol 2014;60:60-62. doi: 10.1016/j.jcv.2014. 01.026 .

[15] Lewis HC, Wichmann O, Duizer E. Transmission routes and risk factors for autochtonous hepatitis E virus infection in Europe: a systematic review. Epidemiol Infect 2010;138:145-166. doi: 10.1017/S0950268809990847.

[16] Nanji AA, French SW. Relationship between pork consumption and cirrhosis. Lancet $1985 ; 1: 681-683$

[17] Dalton HR, Bendall RP, Pritchard C, Henley W, Melzer D. National mortality rates from chronic liver disease and consumption of alcohol and pig meat. Epidemiol Infect 2010;138:174-182. doi: 10.1017/S0950268809990306.

[18] Matsubayashi K, Nagaoka Y, Sakata H, Sato S, Fukai K, Kato T, et al. Transfusion-transmitted hepatitis $\mathrm{E}$ caused by apparently indigenous hepatitis E virus strain in Hokkaido, Japan. Transfusion 2004;44:934940. doi: 10.1111/j.1537-2995.2004.03300.x.

[19] Boxall E, Herborn A, Kocheth G, Pratt G, Adams D, Jiaz S, et al. Transfusiontransmitted hepatitis E in a 'nonhyperendemic' country. Tranfus Med 2006; 16:79-83. doi: 10.1111/j.1365-3148.2006.00652.x.

[20] Colson P, Coze C, Gallian P, Henry M, De Micco P, Tamalet C. Transfusionassociated hepatitis E, France. Emerg Infect Dis 2007;13:648-649. doi: 10. 3201/eid1304.061387. 